\title{
Heart Transplantation in Neonates and Children. Intermediate-Term Results
}

\author{
Estela A zeka, M iguel Barbero-M arcial, Marcelo Jatene, Paulo Roberto Camargo, José O távio C. A uler, \\ Edmar A tik, José A ntonio F. Ramires, Munir Ebaid
}

São Paulo, SP - Brazil

\begin{abstract}
Objective - To assess intermediate-term outcome in children who have undergone orthotopic heart transplantation.

Methods - We carried out a longitudinal and prospective study between October '92 and June '99 comprising 20 patients with ages ranging from 12 days to 7 years (mean of 2.8 years). We employed a double immunosuppression protocol with cyclosporine and azathioprine and induction therapy with polyclonal antithymocyte serum. Survival and complications resulting from the immunosuppression protocol were analyzed.
\end{abstract}

Results - The double immunosuppression protocol and the induction therapy with polyclonal antithymocyte serum resulted in an actuarial survival curve of $90 \%$ and $78.2 \%$ at 1 and 6 years, respectively, with a mean follow-up period of 3.6 years. One patient died due to acute rejection 40 days after transplantation; another patient died 2 years after transplantation due to lymphoproliferative disorder; a third patient died because of primary failure of the graft; and a fourth patient died due to bronchopneumonia. The major complications were as follows: acute rejection, infection, nephrotoxicity, and systemic hypertension. The means of rejection and infection episodes per patient were 2.9 and 3.4, respectively. After one year of transplantation, a slight reduction in the creatinine clearance and systemic hypertension were observed in 7 (38.9\%) patients.

Conclusion - Heart transplantation made life possible for those patients with complex congenital heart diseases and cardiomyopathies in refractory congestive heart failure constituting a therapeutical option for this group of patients in the terminal phase.

Key words: heart transplantation, neonates, children

Instituto do Coração do Hospital das Clínicas-FMUSP and Hospital Sírio Libanês - São Paulo

Mailing address: Miguel Barbero-Marcial - Incor - Divisão de Cirurgia - $2^{\circ}$ S/10 - Av. Dr. Enéas C. Aguiar, 44 - 05403-000 - São Paulo, SP, Brazil
Heart transplantation has made life possible and improved its quality for children with complex congenital heart diseases and cardiomyopathies refractory to the conventional treatment ${ }^{1-3}$.

The first successful orthotopic heart transplantation in a neonate was reported by Bailey et $\mathrm{al}^{4}$. The patient was a newborn with left heart hypoplasia and a good outcome 11 years after the event ${ }^{5}$. Our first transplantation in a patient with left heart hypoplasia was performed in 1992 by Barbero-Marcial et al ${ }^{6}$.

According to the first official report on pediatric heart transplantation by the International Society of Heart and Lung Transplantation $7,3,446$ heart transplantations were performed in 201 medical centers from 1982 to 1996. A higher mortality during the first year, especially in the first 3 months, was observed. The survival curve after the first year showed an annual mortality of $2.5 \%$.

This study aimed to report the intermediate-term results of orthotopic heart transplantation in neonates and children and complications that occurred due to the immunosuppression protocol used.

\section{Methods}

We carried out a prospective and longitudinal study on 20 patients who had undergone orthotopic heart transplantation at INCOR and Hospital Sírio Libanês during the period from October' 92 to June ' 99.

The ages of the patients ranged from 12 days to 7 years (mean of 2.8 years). Distribution according to age ( $<1$ year of age and $>1$ year of age) is shown in figure 1 . Out of the 20 transplanted patients, $11(55.0 \%)$ were males and $9(45.0 \%)$ were females(TableI). Theirweights varied from $3.3 \mathrm{~kg}$ to $20.0 \mathrm{~kg}$, with a mean of $10.41 \mathrm{~kg}$ and a standard deviation of $4.8 \mathrm{~kg}$.

The heart diseases were the following: dilated cardiomyopathy-15(75.0\%) patients; restrictive cardiomyopathy with IIIIV congestive heart failure - $1(5.0 \%)$ patient; and complex congenital heart diseases $-4(20.0 \%)$ patients (Table II). Among those patients under 1 year of age $(75.0 \%)$, the 


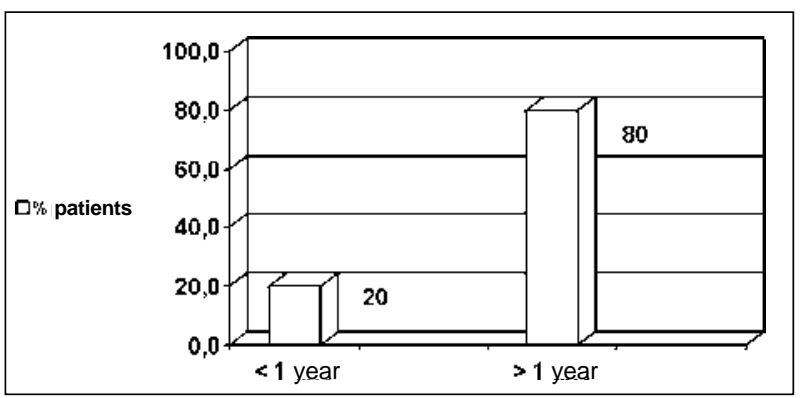

Fig. 1 - Distribution of the 20 patients according to age group.

\begin{tabular}{|c|c|c|c|c|c|c|c|}
\hline \multirow[b]{2}{*}{$\mathrm{N}$} & \multirow[b]{2}{*}{$\begin{array}{c}\text { Age } \\
\text { (days) }\end{array}$} & \multirow[b]{2}{*}{ Sex } & \multirow{2}{*}{$\begin{array}{l}\text { Recipient } \\
\text { LVEF } \\
(\%)\end{array}$} & \multirow[b]{2}{*}{$\mathrm{FC}$} & \multirow[b]{2}{*}{$\begin{array}{l}\text { "Status"/ } \\
\text { priority }\end{array}$} & \multicolumn{2}{|c|}{ Donors } \\
\hline & & & & & & $\begin{array}{c}\text { Age } \\
\text { (days) }\end{array}$ & Sex \\
\hline 1 & 12 & M & & IV & Yes & 21 & M \\
\hline 2 & 916 & M & 19 & III & & 1095 & M \\
\hline 3 & 135 & $\mathrm{~F}$ & & III & & 365 & M \\
\hline 4 & 1946 & M & 13 & IV & & 3650 & M \\
\hline 5 & 477 & M & 19 & IV & & 2190 & $\mathrm{~F}$ \\
\hline 6 & 2167 & $\mathrm{~F}$ & 10 & III & & 3650 & M \\
\hline 7 & 754 & $\mathrm{~F}$ & 12 & III & & 365 & M \\
\hline 8 & 716 & $\mathrm{~F}$ & 16 & IV & & 300 & $\mathrm{~F}$ \\
\hline 9 & 590 & M & & IV & & 365 & M \\
\hline 10 & 1908 & M & 18 & IV & & 2920 & M \\
\hline 11 & 186 & M & 12 & IV & & 300 & M \\
\hline 12 & 907 & M & 8 & IV & Yes & 2555 & M \\
\hline 13 & 1495 & $\mathrm{~F}$ & 16 & IV & & 3285 & $\mathrm{~F}$ \\
\hline 14 & 29 & $\mathrm{~F}$ & & IV & Yes & 10 & $\mathrm{~F}$ \\
\hline 15 & 1066 & $\mathrm{~F}$ & 25 & IV & & 3650 & M \\
\hline 16 & 416 & M & 19 & IV & & 2555 & $\mathrm{~F}$ \\
\hline 17 & 1361 & $\mathrm{~F}$ & 15 & IV & & 2920 & M \\
\hline 18 & 794 & $\mathrm{~F}$ & 20 & IV & & 300 & $\mathrm{M}$ \\
\hline 19 & 1388 & M & & IV & & 2555 & $\mathrm{~F}$ \\
\hline 20 & 2792 & M & & IV & Yes & 2555 & $\mathrm{~F}$ \\
\hline
\end{tabular}

LVEF (\%)- left ventricle ejection fraction by the gated blood pool; Ffemale; M- male; FC - functional class.

\begin{tabular}{|lcc|}
\hline Table II - Indications for heart transplantation in the 20 patients \\
\hline Indications & $\mathrm{N}$ & $\%$ \\
\hline Left heart hypoplasia & 2 & 10.0 \\
Pulmonary atresia with coronary artery-cavitary fistulas & 1 & 5.0 \\
Interventricular septal defect surgically corrected & 1 & 5.0 \\
Dilated cardiomyopathy & 15 & 75.0 \\
Restrictive cardiomyopathy & 1 & 5.0 \\
\hline N-n ${ }^{\circ}$ of patients with indication for heart transplantation; \%-percentage \\
of patients.
\end{tabular}

congenital heart diseases were the most common and the cardiomyopathies occurred in $25.0 \%$. Among those patients older than 1 year, the cardiomyopathies were the most common (93.7\%), and the congenital heart diseases occurred in $6.3 \%$.

In regard to the donors, their ages ranged from 10 days to 10 years, with a mean of 4.9 years and standard deviation of 3.7 years (table I), and their weights ranged from $3.8 \mathrm{~kg}$ to $30.0 \mathrm{~kg}$, with a mean of $16.9 \mathrm{~kg}$ and standard deviation of $8.2 \mathrm{~kg}$. Fourteen $(70.0 \%)$ were males. The diagnosis of cere- bral death resulted more frequently from craniocerebral trauma (12 cases $-60.0 \%)$.

Duration of ischemia varied from $38 \mathrm{~min}$ to $145 \mathrm{~min}$, with a mean of $74.8 \mathrm{~min}$ and standard deviation of $28.4 \mathrm{~min}$.

The criteria of inclusion used for transplantation were the following: patients with complex congenital heart disease or cardiomyopathy in refractory congestive heart failure, or both ${ }^{8}$.

The criteria of exclusion were the following: patients with infection at the time of transplantation; patients with neurological malformations or severe neurological sequelae; patients with alterations in renal function; patients with malformation of the urinary tract and chronic renal failure; patients with severe metabolic disorders; patients with genetic syndromes; premature infants; small-for-gestationalage infants; and patients with pulmonary vascular resistance index higher than 6 units Wood $/ \mathrm{m}^{28,9}$.

Assessment of the potential donors included the following: determination of the blood group to assess compatibility, the donor's weight should be as much as 3 times that of the recipient, cardiovascular and infectious assessment. The cardiovascular criteria for inclusion were the following: the cardiovascular physical examination of the donors, as well as their chest X-rays should be normal; their echocardiogram should have a shortening fraction higher than $25 \%$ and show no heart defects. In regard to infection, the donors should have no infectious process and negative serologies for HIV, hepatitis B and C, and Chagas' heart disease ${ }^{10,11}$.

The cross-match (a test with cells of the donor and plasma of the recipient) was performed in all cases, and a positive result excluded the possibility of using the organ.

Prophylaxis of rejection consisted of immediate use of cyclosporine in the postoperative period. The drug was administered intravenously in a continuous infusion, at the dose of 0.1 to $0.2 \mathrm{mg} / \mathrm{kg} /$ hour; after withdrawal of the endotracheal tube, cyclosporine was administered orally at the initial dose of $20 \mathrm{mg} / \mathrm{kg} / \mathrm{day}$. The drug's dose was controlled according to its serum level through the radioimmunoassay method; cyclosporine level was maintained between 300 and $400 \mathrm{ng} / \mathrm{ml}$ in the first year, and after that, between 150 and $200 \mathrm{ng} / \mathrm{ml}$, according to rejection episodes and assessment of the side effects of the drug.

Azathioprine was administered at the dose of $3 \mathrm{mg} / \mathrm{kg} /$ day, immediately after transplantation. The drug was administered at a dose up to $3 \mathrm{mg} / \mathrm{kg} /$ day in the first year after transplantation; later, it was reduced to $1 \mathrm{mg} / \mathrm{kg} / \mathrm{day}$. Control of the dose was performed according to the number of leukocytes in the peripheral blood and azathioprine was suspended when the count was lower than 3500 cells per $\mathrm{mm}^{3}$.

Methylprednisolone was administered immediately after transplantation at the dose of 125 to $500 \mathrm{mg}$ every 12 hours, according to the child's weight for a period of only 48 hours.

Antithymocyte rabbit or horse serum ${ }^{12}$ was used immediately after transplantation or at the first episode of acute rejection.

After hospital discharge, children with inadequate socioeconomic conditions who had come from other cities and 
states remained for a minimum period of 4 months in the Associação de Assistência à Criança Transplantada do Coração, a philanthropic entity especially created to make transplantation in these patients possible.

Ambulatory follow-up after hospital discharge was performed through sequential assessments twice a week in the first 3 months and, after that, once a month.

The major complications directly related to the transplantation and the immunosuppression protocol were as follows: primary failure of the transplanted heart; right ventricular dysfunction immediately after transplantation; acute rejection; infection; coronary heart disease; tumor; nephrotoxicity; systemic hypertension; hyperlipidemias; and biliary lithiasis ${ }^{13}$.

Primary failure of the transplanted heart was characterized by clinical findings of systemic low output and severe ventricular dysfunction shown on the echocardiogram and whose evolution resulted in death of the patient.

Right ventricular dysfunction was assessed by signs of heart failure and qualitative increase in the right ventricular chambers on the echocardiogram.

The diagnosis of acute rejection was made through clinical, electrocardiographic, and Doppler echocardiographic findings ${ }^{14,15}$.

Endomyocardial biopsy was performed when the signals of acute rejection persisted after the initial treatment with corticosteroids ${ }^{16}$.

Prophylaxis of the infectious processes consisted of respiratory and contact isolation of the patients in the first 3 months after transplantation. Right after transplantation, antibiotics were employed until withdrawal of the drains as was hyperimmune gamma globulin at the dose of $400 \mathrm{mg} / \mathrm{kg} /$ day every other day for a total of 5 doses.

In our study, those infectious episodes considered risky for the life of the child and requiring antimicrobial therapy through oral or intravenous or both viae were analyzed ${ }^{17}$.

Coronary heart disease of the graft was assessed through angiography of the coronary arteries or through the anatomicopathological examination. Coronary angiography was indicated in patients with more than 3 years of evolution.

Lymphoproliferative disorder was defined by the anatomicopathological study of the patients ${ }^{13}$.

Nephrotoxicity of the immunosuppressive drugs was evaluated through measurement of the creatinine clearance in patients more than one year after transplantation ${ }^{13,18}$.

The frequency of systemic hypertension in patients more than one year after transplantation was analyzed ${ }^{19}$.

Hyperlipidemia was regarded as hypercholesterolemia in patients more than one year after transplantation ${ }^{13}$.

The presence of biliary lithiasis was assessed through clinical signs and symptoms of gallstones and through the abdominal ultrasound in patients more than one year after transplantation.

A descriptive analysis of data of the 20 patients was performed comprising demographic data, clinical outcome, and complications. The actuarial survival curve was performed by the Kaplan-Meyer method.

\section{Results}

Out of the 20 patients undergoing heart transplantation, $16(80.0 \%)$ survived. One patient died due to primary failure of the graft 2 days after transplantation. Three patients died after hospital discharge as follows: one due to acute rejection 40 days after transplantation; one due to lymphoproliferative disorder 2 years after transplantation; and another patient due to infection by Pneumocistis carinii, one year after transplantation.

The actuarial survival curve was $90 \%$ and $78.2 \%$ after 1 year and 6 years of transplantation, respectively (fig. 2) with a mean follow-up period of 3.6 years.

Among the 20 patients, the complications directly related to transplantation and immunosuppression were as follows: one patient had primary failure of the graft, dying 48 hours after transplantation, despite the use of assisted circulation; $4(20.0 \%)$ patients had mild ventricular dysfunction; and $2(10.0 \%)$ had moderate to severe ventricular dysfunction secondary to pulmonary hypertension. All patients had a good evolution with administration of vasodilating drugs. Fifty-seven episodes of acute rejection occurred. The number of rejection episodes per patient varied up to 8 with a mean of 2.9 .

Noninvasive diagnostic methods made the diagnosis of acute rejection possible in $91.2 \%$ of the cases. The endomyocardial biopsy was performed only in $5(8.8 \%)$ episodes. Distribution of the episodes of rejection in regard to time is shown in figure 3 .

Sixty-nine infectious events occurred in the 20 patients. Patients had a mean of 3.4 episodes of infection. Distribution of the infectious episodes in regard to the causative agents is shown in figure 4.

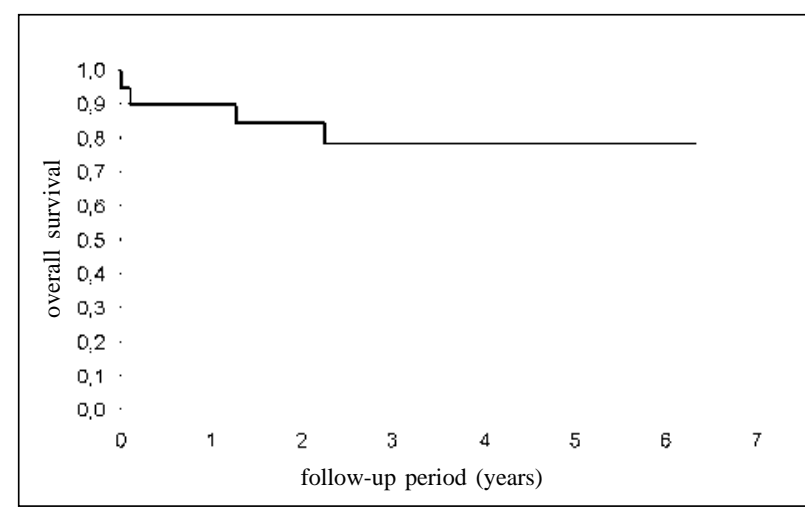

Fig. 2-Survival curve-Kaplan-Meier method - of the 20 patients.

\begin{tabular}{|lccccc|}
\hline $\begin{array}{l}\mathrm{N}^{\circ} \text { of } \\
\text { patients }\end{array}$ & $\begin{array}{c}\mathrm{N}^{\circ} \text { of } \\
\text { deaths }\end{array}$ & $\begin{array}{c}\text { Time } \\
\text { (years) }\end{array}$ & $\begin{array}{c}\mathrm{P} \\
\text { (Survival) }\end{array}$ & $\begin{array}{c}\text { Standard } \\
\text { error }\end{array}$ & $\begin{array}{c}\mathrm{N}^{\circ} \text { of patients } \\
\text { at risk }\end{array}$ \\
\hline & & 1 & 0.900 & 0.067 & 18 \\
& & 2 & 0.847 & 0.081 & 14 \\
& & 3 & 0.782 & 0.098 & 12 \\
& 4 & 4 & 0.782 & 0.098 & 7 \\
& & 5 & 0.782 & 0.098 & 5 \\
& & 6 & 0.782 & 0.098 & 2 \\
\hline
\end{tabular}




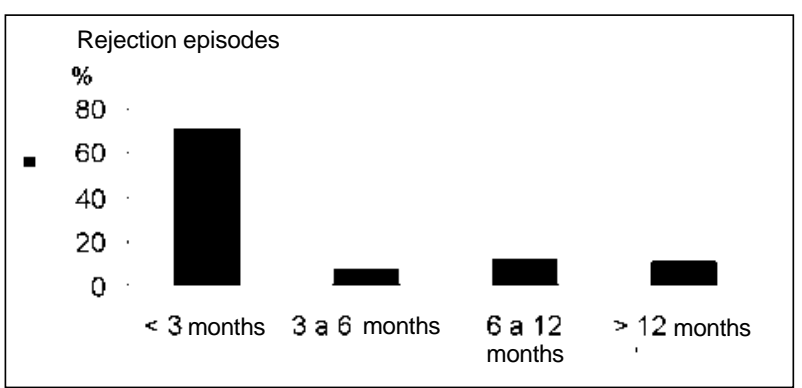

Fig. 3 - Distribution of the frequency of rejection episodes in the 20 patients undergoing heart transplantation in the period of the study.

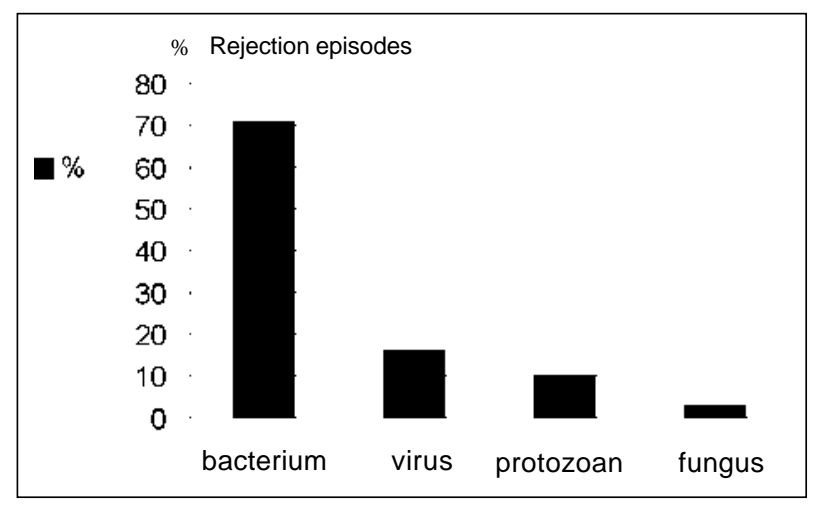

Fig. 4 - Distribution of the frequency of the infectious processes in the 20 patients undergoing heart transplantation according to causative agents.

Angiographic studies performed in 3 patients more than 3 years after transplantation did not show any lesions in the coronary arteries.

Only one $(5.0 \%)$ patient had a tumor 2 years after transplantation with pulmonary nodules revealed on chest tomography. Even though 3 pulmonary biopsies were performed, the definitive diagnosis could only be established after death.

From the 18 patients with more than 1 year of evolution, $7(38.9 \%)$ had a mild decrease in renal function and 7 $(38.9 \%)$ had systemic hypertension.

Out of these 18 patients, $1(5.6 \%)$ had hypercholesterolemia 1 year after transplantation; the angiography, however, was normal.

In regard to gallstones, $2(11.1 \%)$ patients had this complication, and only 1 required surgery, undergoing endoscopic papillotomy and laparoscopic cholecystectomy.

\section{Discussion}

Heart transplantation is the therapy of choice for patients with complex congenital heart diseases and cardiomyopathies with refractory heart failure.

In our study, the types of heart disease with indication for transplantation in children under 1 year of age were similar to those described by Boucek et al ${ }^{7}$. We observed that $75 \%$ of our patients had congenital heart diseases, of which the most common was left heart hypoplasia, and $25 \%$ had cardiomyopathies.
In regard to children older than 1 year of age, $93.7 \%$ had cardiomyopathies and $6.3 \%$ had congenital heart diseases, in accordance with predominance of cardiomyopathies $(51 \%)$ in the first official pediatric report of the International Society of Heart and Lung Transplantation ${ }^{7}$, even though our percentage was higher.

Cardiomyopathy is considered a factor of favorable prognosis ${ }^{7}$. Predominance of this heart disease in children older than 1 year of age in our study may explain the favorable outcome of these children despite their mean age of 2.8 years, considered within the risky age group ${ }^{7}$.

In the clinical trials with double protocols using cyclosporine, azathioprine and induction with antithymocyte rabbit serum, Au et $\mathrm{al}^{20}$ and Fullerton et $\mathrm{al}^{21}$ have reported no death due to rejection. The cause of death was primary dysfunction of the transplanted organ ${ }^{20}$ or right ventricular failure secondary to pulmonary hypertension ${ }^{21}$. However, both studies were conducted in patients older than 5 weeks. Chinnock $^{5}$ carried out the major evolutional study in 299 patients, 92 of whom were under 30 days of age, with actuarial survival curves of $83 \%, 72 \%$, and $66 \%$ at 1,5, and 10 years, respectively. In his study, rejection was the major cause of death, occurring in $8 \%$ of the patients. In our study, $16(80.0 \%)$ out of the 20 patients survived. Four patients died; the first, a newborn with left heart hypoplasia died 40 days after transplantation due to acute rejection; the second patient died 2 years after transplantation due to a tumor; the third patient died due to primary failure of the graft; and the fourth patient died because of pneumonia due to Pneumocistis carinii.

The actuarial survival curve of $90 \%$ and $78.2 \%$ at 1 and 6 years, respectively, was probably the major indicator of favorable clinical outcome in the period of time observed.

In regard to complications, we observed that lower doses and lower levels of the drugs of the immunosuppression protocol one year after transplantation may have contributed to the lower percentage of severe complications observed in this study.

The number of rejection episodes per patient in the studies with double protocols and induction with antithymocyte rabbit serum varied from 0.8 to $2.0^{5,20,21}$. In our study, we observed that the patients had a mean of 2.9 rejection episodes.

Chinnock et al ${ }^{14}$ reported that the diagnosis of acute rejection through endomyocardial biopsy in patients less than 1 year after transplantation was 0.26 . Fullerton et al ${ }^{21}$ performed endomyocardial biopsy only in the cases where the diagnosis of acute rejection was not certain. In that study, because of the risks of the endomyocardial biopsy, it was performed only in $5(8.8 \%)$ out of the 57 episodes of rejection; diagnosis through noninvasive methods was preferred.

Infection is a cause of death according to the official pediatric report of the International Society of Heart and Lung Transplantation ${ }^{7}$. Studies with double protocols show that as many as $4.4 \%$ of patients die due to infection ${ }^{5,20,21}$. We had results similar to those of the study group of the International Society of Heart and Lung Transplantation ${ }^{17}$ in regard to infectious episodes. In our study, infection occurred only in one patient, probably because of the prophylac- 
tic measures adopted right after transplantation and because of early diagnosis and adequate therapy established as soon as the patients had the first signs of fever in an attempt to avoid progression of the infection.

Coronary heart disease is one of the major factors limiting long-term survival in children undergoing heart transplantation. It may occur in all age groups ${ }^{7,22}$. Chinnock ${ }^{5}$ observed that the only risk factor in patients with this disease is the highest number of rejections. In our study, the only patient with this disease evolved with the highest number of rejection episodes of hard therapeutical control.

Tumors represent a severe complication of heart transplantation ${ }^{13}$. Studies of patients receiving the double protocol and therapeutical induction have shown a low incidence of tumors ${ }^{5,20,21}$. In our study, only 1 patient had this complication that evolved with pulmonary nodules, which are a common form of the disease ${ }^{23}$. Nevertheless, despite pulmonary biopsies, the diagnosis was only established after death.

Chronic use of cyclosporine may lead to renal failure because of its nephrotoxicity ${ }^{24}$. The major study on double protocol in children undergoing transplantation with a follow-up period longer than 5 years was carried out by Chinno$\mathrm{ck}^{5}$. This study has shown that most of the patients had a mild reduction in the glomerular filtration rate. In our study, assessment of children with a follow-up period longer than 1 year has shown that 7 (38.9\%) patients evolved with a mild reduction in the creatinine clearance while the others had a normal creatinine clearance. This result may have been more favorable because of the shorter follow-up analyzed.
Hypertension is the major cause of death described in the official pediatric report of the International Society of Heart and Lung Transplantation ${ }^{7}$, and it occurs in approximately $40 \%$ of the patients after a 1-year or 2-year follow-up. In our study on children with a follow-up longer than 1 year, we observed that 7 (38.9\%) children evolved with hypertension, which is a percentage similar to that found by Boucek et al ${ }^{7}$.

Hyperlipidemia, which is another complication associated with immunosuppression described by a number of authors ${ }^{7,26}$, was found in this study only in 1 patient more than 1 year after transplantation. In this case, the coronary angiography was normal; therefore, hypertension was not a potential factor in the development of coronary heart disease.

Gallstones, which is a complication associated with the immunosuppression protocol, has a low incidence of surgery ${ }^{27,28}$. In our study, its frequency was also low. Only 1 patient underwent laparoscopic cholecystectomy.

In conclusion, orthotopic heart transplantation in our service has made intermediate-term survival of children with complex congenital heart disease and cardiomyopathies with refractory functional class III-IV heart failure possible. Therefore, orthotopic heart transplantation is a promising therapeutical option for this group of patients.

\section{Aknowledgments}

We thank Prof. Dr. Geraldo Verginelli for his language assistance.

\section{References}

1. Bailey LL, Wood M, Razzouk A, Arsdell GV, Gundry S. Heart transplantation during the first 12 years of life. Arch Surg 1989; 124: 1221-6.

2. Addonizio LJ. Cardiac transplantation in the pediatric patient. Prog Cardiovasc Dis 1990; 33: 19-34.

3. Starnes VA, Oyer PE, Bernstein D, et al. Heart, heart-lung, and lung transplantation in the first year of life. Ann Thorac Surg 1992; 53: 306-10.

4. Bailey LL, Nehlsen-Cannarella SL, Doroshow RW, et al. Cardiac allotransplantation in newborns as therapy for hypoplastic left heart syndrome. N Engl J Med 1986; 315: 949-51.

5. Chinnock RE. Pediatric heart transplantation at Loma Linda: 1985 to 1996. In: Cecka Terasaki, eds. Clin. Transpl.: Los Angeles, California. UCLA Tissue Typing Laboratory 1996; 1: 145- 51.

6. Barbero-Marcial M, AzekaE, Camargo PR, et al. Transplante cardíaco neonatal e infantil. Arq Bras Cardiol 1996; 67: 165-70.

7. Boucek MM, Novick RJ, Bennett LE, Fiol B, Keck BM, Hosenpud JD. The Registry of the International Society of Heart and Lung Transplantation: First official pediatric report - 1997. J Heart Lung Transplant 1997; 16: 1189-206.

8. Gundry SR, Freedom RM. Cardiac replacement in infants and children: indications and limitations. J Heart Transplant 1991; 10(suppl. 2): 791-801.

9. AzekaE, Auler JOC, Camargo PR, et al. The hemodynamic effects of inhalation of nitric oxide on pulmonary hypertension and its complications in children candidates for heart and heart-lung transplantation. Intensive Care Med 1997; 23(suppl. I): S19.

10. Boucek MM, Kanakriyeh MS, Mathis CM, Trimm III RF, Bailey LL. Cardiac transplantation in infancy: Donors and recipients. J Pediatr 1990; 116: 171-6.

11. Pediatric Heart Transplantation Protocol - Loma Linda International Heart Institute . Loma Linda, 1993.

12. Halloran PF, Miller LW. In vivo immunosuppressive mechanisms. J Heart Transplant 1996; 15: 959-71.
13. Miller LW, Schlant RC, Kobashigawa J, Kubo S, Renlund DG. Task Force 5: Complications. J Am Coll Cardiol 1993; 22: 41-54.

14. Chinnock RE, Baum MF, Larsen RL, Bailey LL. Rejection management and long-term surveillance of the pediatric heart transplant recipient: the Loma Linda Experience. J Heart Lung Transplant 1993; 12(Part 2): S255-S264.

15. Azeka E, Barbero-Marcial M, Soares J, et al. The importance of noninvasive methods on the evaluation of acute rejection in pediatric heart transplantation: $\mathrm{Gal}-$ lium-67 scintigraphy as an option. In: Imai Y, Moma K, eds. Proceedings of the Second World Congress of Pediatric Cardiology and Cardiac Surgery. Nova York: Futura, 1997: 133-6.

16. Billingham ME, Cary NRB, Hammond ME, et al. A working formulation for the standardization of nomenclature in the diagnosis of heart and lung rejection: Heart rejection study group. J Heart Transplant 1990; 9: 587-93.

17. Schowengerdt KO, Naftel DC, Seib PM, et al. Infection after pediatric heart transplantation: Results of a multiinstitutional study. J Heart Lung Transplant 1997; 16: 1207-16.

18. Bennett WM, DeMattos A, Meyer MM, Andoh T, Barry JM. Chronic cyclosporine nephropathy: The Achilles' heel of immunosuppressive therapy. Kidn Int 1996; 50: 1089-1100.

19. National Heart, Lung, and Blood Institute. Report of the second Task Force on blood pressure control in children. Pediatrics 1987;.79: 1-25.

20. AuJ, Gregory JW, Colquhoun IW, etal. Paediatric cardiac transplantation with steroidsparing maintenance immunosuppression. Arch Dis Child 1992; 67: 1262-6.

21. Fullerton DA, Campbell DN, Jones SD, et al. Heart transplantation in children and young adults: early and intermediate-term results. Ann Thorac Surg 1995; 59: 804-12.

22. PahlE, FrickerFJ, Armitage J, et al.Coronary arteriosclerosis in pediatric heart transplant survivors: Limitation of long-term survival. J Pediatr 1990; 116: 177-83.

23. Boyle G; Michaels MG, Webber AS, et al. Posttransplantation lymphoproliferative disorders in pediatric thoracic organ recipients. J Pediatr 1997; 131: 309-13. 
24. Borel JF, Baumann G, Chapman I, et al. In vivo pharmacological effects of ciclosporin and some analogues. Adv Pharmacol 1996; 35: 115-246.

25. Fricker FJ, Armitage JM. Heart and heart-lung transplantation in children and adolescents. In: Adams FH, Emmanouilides GC, Riemenschneider TA, Gutgesell HP, eds. Moss'Heart Disease in Infants, Children, and Adolescents Including the Fetus and Young adult. Baltimore: Williams \& Wilkins, 1995: 495-510.
26. Uzark K, Crowley D, Callow L, Bove E. Hypercholesterolemia after cardiac transplantation in children. Am J Cardiol 1990; 66: 1385-7.

27. Razzouk AJ. Surgical intervention in children after heart transplantation. J Heart Lung Transplant 1993; 12(Part 2): S195-S8.

28. Azeka E. Transplante cardíaco em crianças - Análise clínica evolutiva (Tese de Doutorado). São Paulo: Universidade de São Paulo, 1998: 137p. 